\title{
Regularized multiscale frequency domain full-waveform inversion based on hybrid regularization scheme
}

\author{
Raphael C. L. Rêgo (Federal University of Rio Grande do Norte), Williams A. Lima* (Observatório Nacional), Carlos A. N. da \\ Costa (Federal University of Rio Grande do Norte), Edwin F. Duarte (Federal University of Rio Grande do Norte) and João M. \\ de Araújo (Federal University of Rio Grande do Norte)
}

\section{Copyright 2019, SBGf - Sociedade Brasileira de Geofísica.}

This paper was prepared for presentation at the $16^{\text {th }}$ International Congress of the Brazilian Geophysical Society, held in Rio de Janeiro, Brazil, 19-22 August, 2019.

Contents of this paper were reviewed by the Technical Committee of the $16^{\text {th }}$ International Congress of the Brazilian Geophysical Society and do not necessarily represent any position of the SBGf, its officers or members. Electronic reproduction or storage of any part of this paper for commercial purposes without the written consent of the Brazilian Geophysical Society is prohibited.

\section{Abstract}

Full-waveform inversion is an ill-posed seismic inverse problem with a strong nonlinearity. The most common strategy to mitigate the nonlinearity of full-waveform inversion and make it well-posed is by adopting a multiscale strategy to mitigate its nonlinearity, where the inversion is performed sequentially by moving from low to high frequencies, combined with regularization and preconditioning schemes to drive the inversion toward realistic physical models of the subsurface. In this work, we introduce a hybrid regularization scheme based on the Cauchy and Tikhonov constraint for multiscale frequency domain full-waveform inversion. Our regularization scheme may be a good strategy to recover target in the subsurface composed with smoothly-varying regions and regions with strong contrasts of physical parameters. In addition, we combine our hybrid regularization scheme with an adaptive weighted parameter to relax the requirement of estimating a particular regularization parameter for each inversion stage of a regularized multiscale frequency domain fullwaveform inversion. Numerical examples carried out with a synthetic model demonstrates that the inverted model by our scheme is a solution between the inverted models by full-waveform inversion using Tikhonov and Cauchy constraint.

\section{Introduction}

Full-waveform inversion (FWI) technique aims to recover detailed medium parameters of the subsurface by a data fitting procedure based on fitting the measured with modeled full wavefields at the receiver positions (Lailly, 1983; Tarantola, 1984; Virieux and Operto, 2009). However, it is a difficult process to recover a realistic set of medium properties of the subsurface by FWI. This is mainly because FWI is an ill-posed/conditioned inverse problem with a strong nonlinearity (Virieux and Operto, 2009; Alkhalifah, 2014) - meaning that its objective function can have multiple local-minima and that its solution is nonunique and/or unstable.

One way to mitigate the nonlinearity of $\mathrm{FWI}$ is by making the inversion in stages, starting the process with the part of the observed data that has a more linear relation with the model and using the inversion result of this stage as an initial guess to the next stage, in one way where the degree of nonlinearity of the observed data used in each inversion stage increases with stages. As for example, the multiscale inversion strategy (Kolb et al., 1986; Pica et al., 1990; Bunks et al., 1995), where the inversion is performed sequentially by moving from low to high frequencies, using the inversion result of one frequency or group of frequencies as an initial guess to the next. The issue of stability and non-uniqueness of FWI can be addressed by incorporating regularization/preconditioning schemes in its formulation, which helps to drive the inversion toward a realistic model of the subsurface. Thus, a formulation of $\mathrm{FWI}$ as a regularized multiscale inversion scheme increases the chance of finding a stable solution that represents the global minimum valley of the inversion problem. However, in a regularized multiscale inversion scheme, each inversion stage is a new inversion problem, and if this inversion scheme is formulated with an additive regularization term, it is necessary to set an appropriate regularization parameter for each inversion stage, which is a complicated process.

Regularization schemes are commonly divided into two classes: regularization schemes to impose smooth character on the solution of the inversion problem, like Tikhonov constraint (Tikhonov and Arsenin, 1977; BerteteAguirre et al., 2002) and regularization schemes to impose sparseness on the final inversion solution, like total variation constraint (Bertete-Aguirre et al., 2002) or Cauchy constraint (Guitton, 2012). However, constraints to impose smooth feature on the inversion solution are good options to recover smooth models and constraints to impose sparseness are suitable to recover models with strong discontinuities, but in general, the physical models, like geological models of the subsurface, are a combination of smoothly-varying regions with regions with strong contrasts of physical parameters. There are in the literature some strategies in the direction of hybrid regularization schemes, which combining softness and sparseness features in order to recover physical models composed with smoothly-varying regions and regions with strong contrasts of physical parameters. For example, a hybrid regularization scheme is proposed by Gholami and Hosseini (2013), where the hybrid regularization scheme is based on Tikhonov and total variation regularization for reconstruction of piecewise-smooth signals, the others hybrid regularization scheme are proposed by Song et al. (2015), where the regularization method combining Tikhonov with total variation for application in electrical resistance tomography, and by Aghamiry et al. (2018) where the hybrid regularization scheme is formulated for 
frequency domain $\mathrm{FWI}$ based on wavefield reconstruction (van Leeuwen and Herrmann, 2013).

In order to recover targets in the subsurface composed with smoothly-varying regions and regions with strong contrasts of physical parameters, we introduce in this work a regularized multiscale frequency domain FWI based on hybrid regularization scheme, where the hybrid regularization combining Tikhonov with Cauchy constraint. Furthermore, as our regularized FWI formulation uses the hybrid regularization as an additive regularization term, we combine our inversion scheme with an adaptive weighted parameter to relax the requirement of estimating a particular regularization parameter for each inversion stage. We demonstrate the effectiveness of our scheme by numerical examples performed with synthetic data modeled with a two-dimensional (2D) P-wave velocity model.

\section{Methodology}

We have formulated our approach as a gradient based FWI scheme (Lailly, 1983; Tarantola, 1984; Pratt et al., 1998; Virieux and Operto, 2009) adopting the $l_{2}$ norm $\|\cdot\|_{2}$ (Tarantola, 1987; Wang, 2017) for the data misfit term and an additive regularization term, where the regularization term is a hybrid regularization scheme combining Tikhonov with Cauchy constraint. In addition, we have used the theory of adjoint-state method (Plessix, 2006) to compute the gradient of the objective function.

\section{The regularized objective function}

As each inversion stage from a multiscale FWI scheme is a new inversion problem, we attempt to minimize the following regularized objective function for each inversion stage $s$ :

$$
\begin{aligned}
\Phi_{s}(\mathbf{m}) & =\frac{1}{2} \sum_{i=N_{\omega}^{s, f}}^{N_{s}^{s, l}} \sum_{j=1}^{N_{j}} W_{s}\left\|\Gamma_{j} \mathbf{u}_{j}\left(\mathbf{m}, \omega_{i}\right)-\mathbf{d}_{j}^{(o b s)}\left(\omega_{i}\right)\right\|_{2}^{2} \\
& +\mu_{s} \phi\left(\mathbf{m}, \eta_{s}\right),
\end{aligned}
$$

such that at every iteration, the monochromatic acoustic wavefield $\mathbf{u}_{j}\left(\mathbf{m}, \omega_{i}\right) \in \mathbb{C}^{M \times 1}$, over the complex field $\mathbb{C}(M$ indicates the number of grid points of the full-computational domain) and generated by a source $j$, is obtained by the solution of the discretized acoustic wave equation in the frequency-space domain (Marfurt, 1984):

$$
\mathbf{B}\left(\mathbf{m}, \omega_{i}\right) \mathbf{u}_{j}\left(\omega_{i}\right)=\mathbf{F}_{j}\left(\omega_{i}\right) .
$$

Where $\mathbf{B}\left(\mathbf{m}, \omega_{i}\right) \in \mathbb{C}^{M \times M}$ is the monochromatic impedance matrix built from the finite-difference $(F D)$ approximation of the acoustic wave-equation in the frequency-space domain (da Costa et al., 2019) with absorbing boundary condition Perfectly Matching Layer (PML) (Bérenger, 1994). The vector $\mathbf{m} \in \mathbb{R}^{M \times 1}$, over the real field $\mathbb{R}$, represents the $\mathrm{P}$ wave velocity model discretized on a grid over the fullcomputational domain and $\omega_{i}$ represents the ith angular frequency sample, where for simplicity, we are using $\omega_{i} \doteq i \Delta \omega$, where $\Delta \omega$ represents the angular frequency sampling interval. Finally, the monochromatic column vector $\mathbf{F}_{j}\left(\omega_{i}\right) \in \mathbb{C}^{M \times 1}$ represents a source $j$. In the first part of equation (1) (data misfit term), $\mathbf{d}_{j}^{(o b s)}\left(\omega_{i}\right) \in \mathbb{C}^{N_{r} \times 1}$ represents the observed data set $\left(N_{r}\right.$ indicates the total number of receivers) generated by a source $j$. The matrix $\Gamma_{j} \in \mathbb{R}^{N_{r} \times M}$ is a linear operator that extracts, for each source $j$, the values of the modeled wavefield at the receiver positions. The parameters $N_{\omega}^{s, f}$ and $N_{\omega}^{s, l}$ indicate the first and last frequency sample, respectively, used in each inversion stage $s$, with $N_{\omega}^{0} \leq N_{\omega}^{s, f} \leq N_{\omega}^{s, l} \leq N_{\omega}^{\max }$, where $N_{\omega}^{0}$ and $N_{\omega}^{\max }$ represent the first and last frequency sample available in the observed data set, respectively, and $N_{j}$ represents the total number of sources. Finally, the parameter $W_{s}$ is an adaptive weighting parameter (dimensionless), which will be introduced in details later. In the second part of equation (1) (regularization term), the parameter $\mu_{s}$ is a trade-off parameter (regularization parameter) that balances the data misfit term and the influence on the inversion result of the regularization term for each inversion stage $s$, and $\phi\left(\mathbf{m}, \eta_{s}\right)$ represents the hybrid regularization term: a combination of the Tikhonov with Cauchy constraint. The parameter $\eta_{s}$ is a dimensionless weighting parameter which controls the influence of the Tikhonov and Cauchy constraint on the final result of the inversion process. Note that this parameter may be different for each inversion stage.

\section{The adaptive weighted parameter}

As was discussed by Guitton (2012) in a regularized multiscale FWI scheme in the time-space domain, a more intuitive way to estimate $\mu_{s}$ is through an user-defined dimensionless ratio $R_{S}$, which is given by the ratio between the initial value of the data misfit term and the regularization term:

$$
\mu_{s}=\frac{\sum_{i=N_{\omega}^{s, f}}^{N_{s}^{s, l}} \sum_{j=1}^{N_{j}}\left\|\Gamma_{j} \mathbf{u}_{j}\left(\mathbf{m}, \omega_{i}\right)-\mathbf{d}_{j}^{(o b s)}\left(\omega_{i}\right)\right\|_{2}^{2}}{R_{s} \phi\left(\mathbf{m}, \eta_{s}\right)} .
$$

The subscript $s$ in the regularization parameter $\mu_{s}$ indicates that, in principle, we should estimate a specific value of $\mu_{s}$ for each inversion stage $s$, which is a difficult process. On the other hand, we have numerically observed that to use the same regularization parameter for all inversion stages may work well if the initial value of the data misfit term is approximately the same for all stages. However, we have also observed that the initial value of the data misfit term in a multiscale frequency domain FWI can be very different from one stage to another. In order to relax the requirement of estimating a particular regularization parameter for each inversion stage, we inserted a weighting parameter $W_{s}$ in the data misfit term of the regularized objective function in order to keep the initial value of the data misfit the same for all inversion stages. Note that this parameter does not change the topology of the data misfit term. This weighting parameter is adapted for each inversion stage, where its value is estimated automatically during the inversion process from the ratio between the initial value of data misfit term of the first and current inversion stage:

$$
W_{s}=\frac{\sum_{i=N_{\omega}^{1, f}}^{N_{1, l}^{1, l}} \sum_{j=1}^{N_{j}}\left\|\Gamma_{j} \mathbf{u}_{j}\left(\mathbf{m}, \omega_{i}\right)-\mathbf{d}_{j}^{(o b s)}\left(\omega_{i}\right)\right\|_{2}^{2}}{\sum_{i=N_{\omega}^{s, f}}^{N_{\omega}^{s, l}} \sum_{j=1}^{N_{j}}\left\|\Gamma_{j} \mathbf{u}_{j}\left(\mathbf{m}, \omega_{i}\right)-\mathbf{d}_{j}^{(o b s)}\left(\omega_{i}\right)\right\|_{2}^{2}} .
$$

Note that if we use the same $\mu_{s}$ for all inversion stages, this does not imply that $R_{s}$ will be the same for all stages, because the latter also depends on the regularization term, which, in general, has a different value in each inversion stage. 


\section{The hybrid regularization scheme}

Based on the features of Cauchy and Tikhonov constraint, we propose a hybrid regularization scheme formulated as a combination of these two constraints, given by:

$$
\begin{aligned}
\Phi\left(\mathbf{m}, \eta_{s}\right) & =\eta_{s} \phi^{(C)}(\mathbf{m})+\left(1-\eta_{s}\right) \phi^{(T)}(\mathbf{m}) \\
& =\eta_{s} \frac{W_{\phi}}{2} \sum \log \left(1+\frac{|\nabla m|^{2}}{\lambda^{2}}\right) \\
& +\left(1-\eta_{s}\right) \frac{1}{2} \sum \frac{|\nabla m|^{2}}{\beta^{2}} .
\end{aligned}
$$

Where the first term is the Cauchy constraint of first order (where $\nabla$ represents the standard spatial differential operator) and the second term is the Tikhonov constraint of first order. The parameter $\beta$ in the Tikhonov stabilizer make it dimensionless and the parameter $\lambda$ in the Cauchy term is used to make it dimensionless and to control the sparseness imposed by this constraint in the inversion solution (Guitton, 2012; Wang, 2017). As was pointed out previously, the parameter $\eta_{s}$ is a dimensionless weighting parameter that balances the influence of the Tikhonov and Cauchy constraint on the solution of inversion problem. Thus, the choice of this parameter is very important in our regularized scheme, because it will determine the character of the solution of the inversion problem. As in theory, FWI can estimate quantitative models of the subsurface with high resolution, we have imposed high weight to Cauchy constraint and using the Tikhonov constraint to refine the influence of Cauchy constraint on the inversion result. Finally, as the value of Cauchy and Tikhonov regularization may be different in each iteration, we have introduced the parameter $W_{\phi}$ to make sure that both constraints have the same value and that the balance between them is controlled exclusively by the parameter $\eta_{s}$. We use the following equilibrium equation to estimate the parameter $W_{\phi}$ for every iteration:

$$
1=\frac{\phi^{(T)}(\mathbf{m})}{W_{\phi} \phi^{(C)}(\mathbf{m})} .
$$

Note that in this equation we are considering that the constraints are different of zero.

\section{The strategies of inversion}

In order to attenuate the degree of nonlinearity of FWI, we have adopted the multiscale inversion process by inversion of a group of frequencies in each inversion stage $s$, where each inversion stage is determined by $N_{\omega}^{s, f}$ and $N_{\omega}^{s, l}$, which represents the first and last frequency used in each group of frequencies inverted simultaneously, respectively. In this strategy, the inversion is performed sequentially by moving from low to high frequencies, using the inversion result of one group of frequencies as an initial guess to the next one.

To set the parameter $\eta_{s}$, we first divided all inversion stages in three big groups (here, we are using nearly the same number of inversion stages for each group), such that for each group, we set a specific value for $\eta_{s}[0.95,0.85,0.75]$, where we have started with high value and decrease it for the next two groups, but always using $\eta_{s} \geq 5$.

For inversion performed using the hybrid, Tikhonov and Cauchy regularization, we use $R_{S}=50.0, R_{S}=25.0$ and $R_{S}=3.0$ to determine the regularization parameter in the first stage of each big group used to set the value of $\eta_{s}$, respectively. This means that we have used only three different regularization parameters during all inversion stages.

To perform some numerical examples, we have adopted the Quasi-Newton optimization method L-BFGS-B (Nocedal, 1980; Nocedal and Wright, 2006) to find the velocity model $\mathbf{m}$ that minimizes the regularized objective function in each inversion stage.

\section{Numerical examples}

The effect of our regularized inversion scheme will be demonstrated on noise-free synthetic seismic data set, which was modeled with a code based on the classic FD approximation of 9 points of the 2D acoustic fullwave equation in the frequency-space domain. In addition, this code is constructed with PML to attenuate the boundary reflections from the sides of computational domain. Furthermore, as our FWI code requires as input the observed data in the time domain and our modeling is performed in the frequency-space domain, we applied the Fastest Fourier Transform in the West (FFTW) (Frigo and Johnson, 2005) on the modeled waveforms to get them in the time-space domain.

\section{The modeling of the observed data set}

In this work, we are claiming that our regularized inversion scheme is suitable to recover target in the subsurface composed with smoothly-varying regions and regions with strong contrasts of physical parameters. Thus, to generate the observed data set to test our scheme, we constructed a P-wave velocity model (which we named BUJA2019 velocity model), displayed in Figure 1(a), which has both features: smoothly-varying regions and regions with strong velocity contrasts. In addition, as density distribution we used a homogeneous density model with $1200.0 \mathrm{~kg} / \mathrm{m}^{3}$. The model cover an extension of $6.0 \mathrm{~km}$ in the lateral direction and $2.0 \mathrm{~km}$ in depth, with a regular discretization of $0.008 \mathrm{~km}$. We used 76 sources distributed just below the surface $(z=0.048 \mathrm{~km})$, with increments of $0.08 \mathrm{~km}$, and a fixed set of 376 receivers distributed in a line in the lateral direction at $z=0.096 \mathrm{~km}$, equally spaced with $0.016 \mathrm{~km}$. We used the Ricker pulse form as wavelet with $10.5 \mathrm{~Hz}$ peak frequency (maximum frequency around $31.5 \mathrm{~Hz}$ ). In Figures 1(b) and 1(c), we displayed two shotgathers from this modeling, from sources positioned at $0.96 \mathrm{~km}$ and 4.96 $\mathrm{km}$ in the lateral direction, respectively. In this modeling we are not considering a free-surface.

\section{The waveform inversion results}

Here, an inversion process by FWI (i.e., a complete FWI result) consists of an inversion of a frequency bandwidth of $27.0 \mathrm{~Hz}$, from $2.5 \mathrm{~Hz}$ up to $29.5 \mathrm{~Hz}$, divided in 14 inversion stages carried out successively (with a maximum of 100 iterations per group of frequencies inverted). For each inversion stage, we have used a coverage of 2.0 Hz, i.e., $N_{\omega}^{s, f}-N_{\omega}^{s, f}=2.0 \mathrm{~Hz}$, with increments of $0.5 \mathrm{~Hz}$ into each group of frequencies inverted. In addition, we have considered that the homogeneous water layer, the maximum and minimum velocity of the model and the wavelet are known as an a priori.

For this example, we set $\lambda=10 \mathrm{~m} / \mathrm{s}$ and $\beta=\sqrt{10} \mathrm{~m} / \mathrm{s}$. These parameters have units of velocity because the 


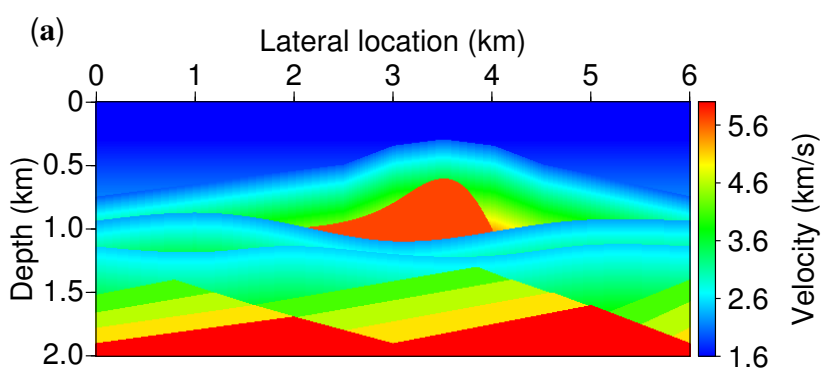

(b)

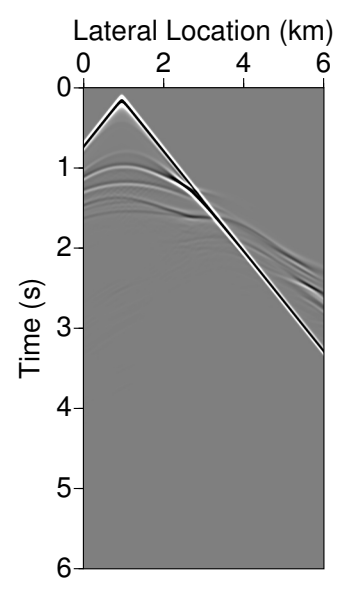

(c)

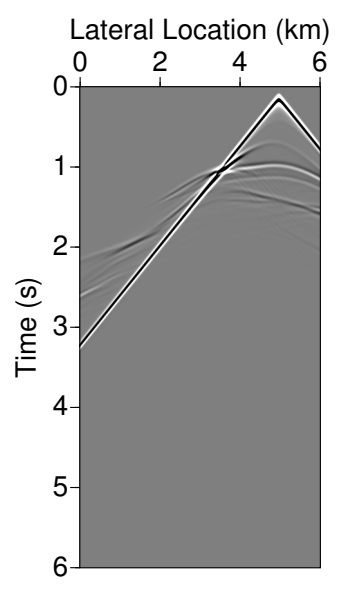

Figure 1: (a) P-wave velocity model used to model the observed synthetic data set. (b) Shotgather generated by a source positioned at $x=4.6 \mathrm{~km}$. (c) Shotgather generated by a source positioned at $x=4.96 \mathrm{~km}$.

derivatives of the velocity model in the regularization term are not divided by $d x$ or $d z$.

We used as initial P-wave velocity model for inversion a smoothed version of the true model without the salt body, displayed in Figure 2(a), which was obtained with the program smooth2 (using $r 1=r 2=30$ ) of the Seismic Unix (Cohen and Stockwell, 2008) software package. Note that this initial model has a good cinematic information, which is an a priori information about the subsurface that can be estimate by Wave-equation Migration Velocity Analysis (Biondi and Sava, 1999; Sava and Biondi, 2004a,b; Alkhalifah and Wu, 2017), for example.

We begin our numerical examples showing that the initial value of data misfit term of the regularized objective function may be very different from an inversion stage to another, and that this implies that we should estimate a specific regularization parameter for each inversion stage, which is a very difficult process. To show this, we performed an inversion process by FWI without regularization, where the result is displayed in Figure 2(b). We can see from this inversion result that the inversion process works well. In Table 1, we show the normalized initial value of the data misfit term of this inversion process of each inversion stage carried out. For this example, the difference between the initial value of the data misfit term of the first and second inversion stage is more than $80 \%$. In addition, we can see that all initial value of the data misfit
Table 1: Normalized initial value of the objective function of each inversion stage

\begin{tabular}{r|l}
\hline Stages & Normalized initial value \\
\hline 1 & 1.0 \\
2 & 0.176172506 \\
3 & 0.158033488 \\
4 & 0.169412255 \\
5 & 0.176186259 \\
6 & 0.084483547 \\
7 & 0.050677233 \\
8 & 0.020439166 \\
9 & 0.008954912 \\
10 & 0.002821997 \\
11 & 0.000681794 \\
12 & 0.000222215 \\
13 & 0.000055216 \\
14 & 0.000008844 \\
\hline
\end{tabular}

term are different from one another. This indicates that to impose some features on the inversion solution by an additive regularization term, it can be necessary a specific value of the trade-off parameter, which balances the data misfit and the influence on the inversion result of the regularization term, for each inversion stage. This makes sense because each inversion stage is a new inversion problem. Because that, in this work we introduce the parameter $W_{s}$ in order to relax the requirement of a specific regularization parameter for each inversion stage.

To show the effectiveness of this parameter, we carried out the inversion process by FWI using the Tikhonov and Cauchy constraint as an additive regularization term. In Figure 3 we displayed the inversion results using the Tikhonov regularization combined $W_{s}$ (Figure $3 a$ ) and without this parameter (Figure $3 b$ ). We can see from these results that the inversion result without using the parameter $W_{s}$ was strongly smoothed, as expected. In Figure 4, we displayed the inversion results using Cauchy regularization combined $W_{s}$ (Figure $4 \mathrm{a}$ ) and without this parameter (Figure 4b). We can see in this case that the inversion result without using the parameter $W_{s}$ was strongly affected by the regularization term.

Finally, in Figure 5 we displayed the regularized FWI result using the hybrid regularization scheme. As expected, the inversion result is a solution between the inverted models by FWI using Tikhonov and Cauchy constraint.

\section{Conclusions}

In this work, we discuss a hybrid regularization scheme for multiscale frequency domain FWI. This scheme can be a good strategy to recover target in the subsurface composed with smoothly-varying regions and regions with strong contrasts of physical parameters.

We also introduce a strategy to relax the requirement of a specific regularization parameter for each inversion stage of a regularized multiscale frequency domain FWI with an additive regularization term. This strategy is based on the observation that only one regularization parameter may work well for all inversion stages if the initial value of the data misfit term is approximately the same for all stages. 


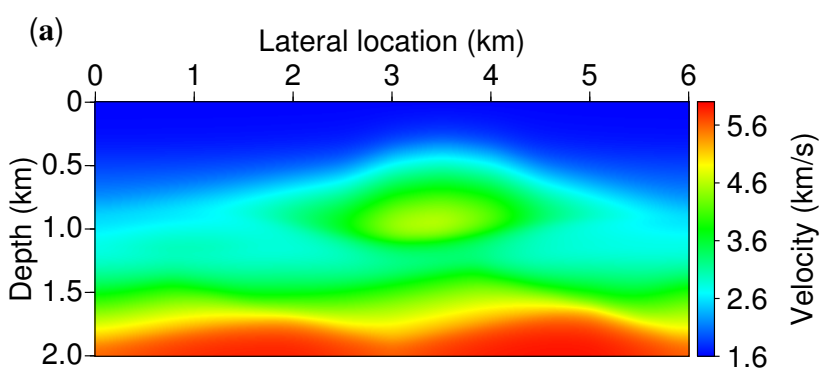

(b) Lateral location $(\mathrm{km})$

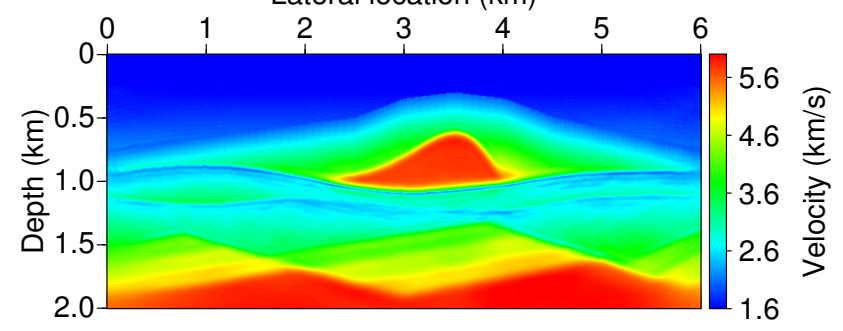

Figure 2: (a) Initial P-wave velocity model to start the inversion process. (b) Inversion result by FWI after 14 groups of frequencies inverted successively, from $2.5 \mathrm{~Hz}$ up to $29.5 \mathrm{~Hz}$, using maximum of 100 iterations per group of frequencies inverted.

(a)

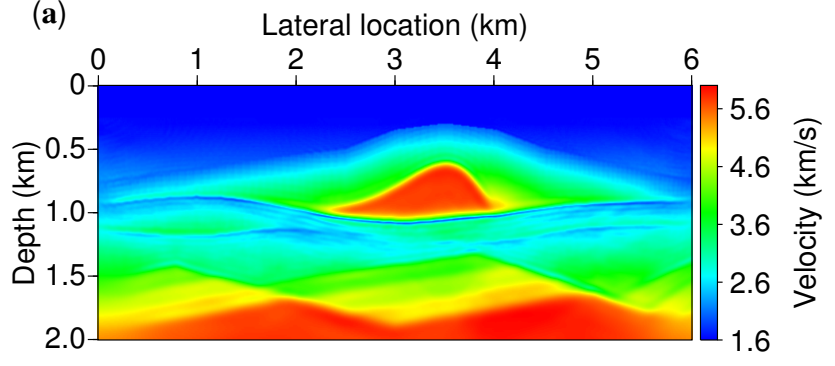

(b)

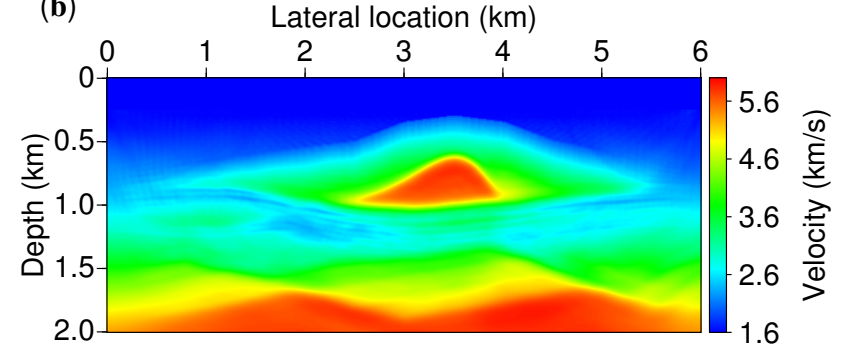

Figure 3: Inversion result by regularized FWI with Tikhonov regularization after 14 groups of frequencies inverted successively, from $2.5 \mathrm{~Hz}$ up to $29.5 \mathrm{~Hz}$, using maximum of 100 iterations per group of frequencies inverted. (a) Using the parameter $W_{s}$. (b) Without using $W_{s}$.

Thus, in order to use the same regularization parameter in all stages, we inserted a weighting parameter within the objective function in order to keep the initial value of the data misfit term the same for all inversion stages.

We showed numerically that the inversion solution by the regularized multiscale frequency domain FWI using

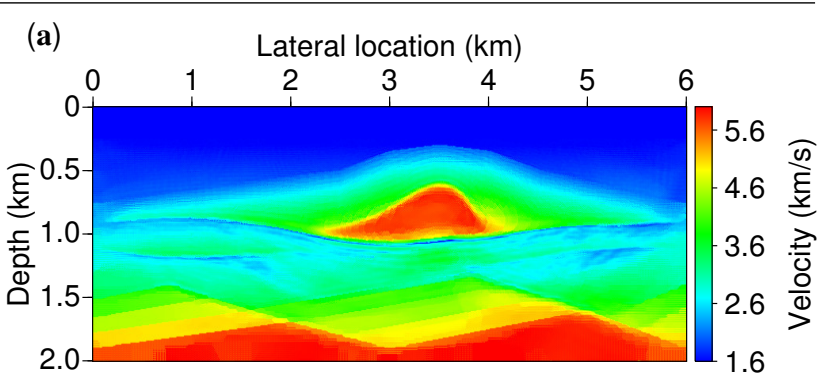

(b) Lateral location $(\mathrm{km})$

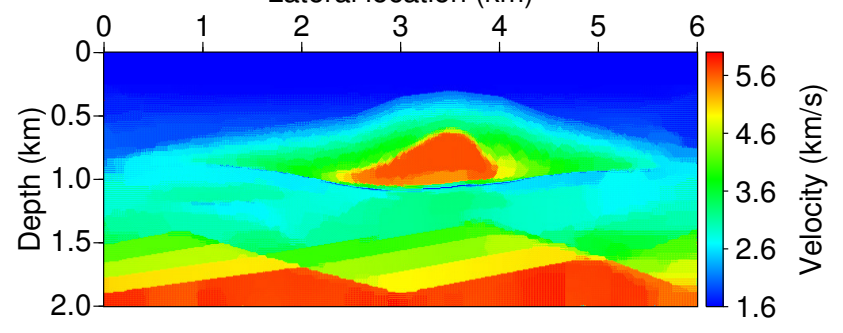

Figure 4: Inversion result by regularized FWI with Cauchy regularization after 14 groups of frequencies inverted successively, from $2.5 \mathrm{~Hz}$ up to $29.5 \mathrm{~Hz}$, using maximum of 100 iterations per group of frequencies inverted. (a) Using the parameter $W_{s}$. (b) Without using $W_{s}$.

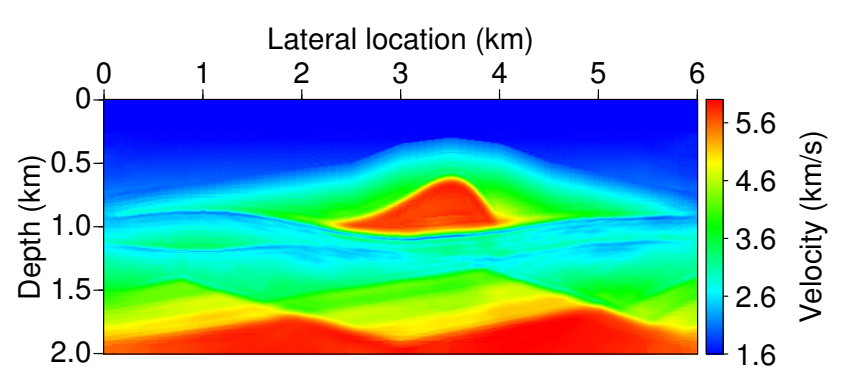

Figure 5: Inversion result by regularized FWI with hybrid regularization after 14 groups of frequencies inverted successively, from $2.5 \mathrm{~Hz}$ up to $29.5 \mathrm{~Hz}$, using maximum of 100 iterations per group of frequencies inverted.

our hybrid regularization scheme is a solution between the inverted models by FWI using Tikhonov and Cauchy constraint. In addition, we showed numerically that the adaptive weighted parameter for the data misfit term can be a good strategy to relax the requirement of a specific regularization parameter for each inversion of a regularized multiscale frequency domain $\mathrm{FWI}$ with an additive regularization term.

\section{ACKNOWLEDGMENTS}

The authors gratefully acknowledge support from Shell Brazil through the "New Methods for Full-Waveform Inversion" project at Federal University of Rio Grande do Norte and the strategic importance of the support given by ANP through the R\&D levy regulation. 


\section{References}

Aghamiry, H. S., Gholami, A., and Operto, S., 2018, Hybrid tikhonov + total-variation regularization for imaging large-contrast media by full waveform inversion: 88th SEG meeting, California, 1253-1257.

Alkhalifah, T., and Wu, Z., 2017, Migration velocity analysis using pre-stack wave fields: Geophysical Prospecting, 65 , no. 3, 639-649.

Alkhalifah, T. A., 2014, Full waveform inversion in an anisotropic world: Where are the parameters hiding ?: EAGE.

Bérenger, J., 1994, A perfectly matched layer for the absorption of electromagnetic waves: Journal of Computational Physics, 114, 185-200.

Bertete-Aguirre, H., Cherkaev, E., and Oristaglio, M., 2002, Non-smooth gravity problem with total variation penalization functional: Geophysical Journal International, 149, no. 2, 499-507.

Biondi, B., and Sava, P., 1999, Wave-equation migration velocity analysis: 69th SEG meeting, Houston, 17231726.

Bunks, C., Saleck, F. M., Zaleski, S., and Chavent, G., 1995, Multiscale seismic waveform inversion: Geophysics, 60, no. 5, 1457-1473.

Cohen, J. K., and Stockwell, J. J. W., 2008, Cwp/su: Seismic unix release no. 41: An open source software package for seismic research and processing,center for wave phenomena, colorado school of mines:.

da Costa, C. A. N., Costa, J. C., Medeiros, W. E., Verschuur, D. J., and Soni, A. K., 2019, Targetlevel waveform inversion: a prospective application of the convolution-type representation for the acoustic wavefield: Geophysical Prospecting, 67, no. 1, 69-84.

Frigo, M., and Johnson, S. G., 2005, The Design and Implementation of FFTW3: Proceedings of the IEEE, 93, no. 2, 216-231.

Gholami, A., and Hosseini, S. M., 2013, A balanced combination of tikhonov and total variation regularizations for reconstruction of piecewise-smooth signals: Signal Processing, 93, 1945-1960.

Guitton, A., 2012, Blocky regularization schemes for fullwaveform inversion: Geophysical Prospecting, 60, 870884.

Kolb, P., Collino, F., and Lailly, P., 1986, Pre-Stack Inversion of a 1-D Medium: Proceedings of the IEEE, 74, no. 3 , 498-508.

Lailly, P., 1983, The seismic inversion problem as a sequence of before stack migrations: Conference on Inverse Scattering, Theory and Application, 206-220.

Marfurt, K. J., 1984, Accuracy of finite-difference and finite-element modeling of the scalar and elastic wave equations: Geophysics, 49, no. 5, 533-549.

Nocedal, J., and Wright, S. J., 2006, Numerical optimization: Springer.
Nocedal, J., 1980, Updating quasi-newton matrices with limited storage: Mathematics of Computation, 35, no. 151, 773-782.

Pica, A., Diet, J. P., and Tarantola, A., 1990, Nonlinear inversion of seismic reflection data in a laterally invariant medium: Geophysics, 55, no. 3, 284-292.

Plessix, R. E., 2006, A review of the adjoint-state method for computing the gradient of a functional with geophysical applications: Geophysical Journal International, 167, no. 2, 495-503.

Pratt, R. G., Shin, C., and Hicks, G. J., 1998, GaussNewton and full Newton methods in frequency-space seismic waveform inversion: Geophysical Journal International, 133, no. 2, 341-362.

Sava, P., and Biondi, B., 2004a, Wave-equation migration velocity analysis. I: Theory: Geophysical Prospecting, 52, no. 3, 593-606.

Sava, P., and Biondi, B., 2004b, Wave-equation migration velocity analysis. II: Subsalt imaging examples: Geophysical Prospecting, 52, no. 3, 607-623.

Song, X., Xu, Y., and Dong, F., 2015, A hybrid regularization method combining tikhonov with total variation for electrical resistance tomography: Flow Measurement and Instrumentation, 46, 268-275.

Tarantola, A., 1984, Inversion of seismic reflection data in the acoustic approximation: Geophysics, 49, no. 8, (8), 1259-1266.

Tarantola, A., 1987, Inverse problem theory: Methods for data fitting and model parameter estimation: Elsevier Science Publication Company, Inc.

Tikhonov, A. N., and Arsenin, V. Y., 1977, Solutions of illposed problems: John Wiley \& Sons.

van Leeuwen, T., and Herrmann, F. J., 2013, Mitigating local minima in full-waveform inversion by expanding the search space: Geophysical Journal International, 195, 661-667.

Virieux, J., and Operto, S., 2009, An overview of full-waveform inversion in exploration geophysics: Geophysics, 74, no. 6, WCC1-WCC26.

Wang, Y., 2017, Seismic Inversion: Theory and Applications: John Wiley \& Sons, Ltd. 